of the work of public health emergency and major event security and has been integrated into the health emergency response mechanism. As shown by the discussion above, our research generally demonstrates that there is a shortage of knowledge about emergency risk communication, a lack of positive attitude toward it, and a lack of capability of health emergency response staff in Chongqing, China. Ambiguity in responsibility has always been a prominent issue that hinders further improvement in emergency preparedness capacity for public health works in China. Furthermore, emergency risk communication work is in the beginning stages. Therefore, it is urgent to reinforce training to enhance people's awareness of emergency and their response capacity.

Our study did have a bias because it relied on a non-validated questionnaire and capacity and knowledge were self-reported, which are closely related to personal feeling. Even so, we believe that this theme will encourage more scholarly engagement in the study of this problem. At the same time, we hope that these findings are helpful in providing the basis for relevant departments to formulate policies and to promote the development of emergency risk communication in the People's Republic of China.

\section{About the Authors}

School of Public Health and Management, Center for Research of Medicine $\mathcal{E}$ Social Development, the Innovation Center for Social Risk Governance in Health,
Chongqing Medical University, Chongqing, China (Lu Tang, Yan Zhang, Kaocong Tian, Jingfu Qiu); Chongqing Municipal Health and Family Planning Commission, Chongqing, China (Dianguo Xing); Chongqing Centers for Disease Control and Prevention, Chongqing, China (Jun Sun).;

Correspondence and reprint requests to Professor Yan Zhang, Chongqing Medical University, 1 Yixueyuan Road, Yuzhong District, Chongqing, 400016, China (e-mail:cqmudzy@163.com).

Published online: March 29, 2016.

\section{REFERENCES}

1. Centers for Disease Control and Prevention. Basic CERC Quick Guide. http://emergency.cdc.gov/cerc/resources/pdf/cerc_guide_basic.pdf. Published 2008. Accessed March 9, 2016.

2. Shimura T, Yamaguchi I, Terada H, et al. Public health activities for mitigation of radiation exposures and risk communication challenges after the Fukushima nuclear accident. J Radiat Res. 2015;56(3):422-429. doi: $10.1093 / \mathrm{jrr} / \mathrm{rrv} 013$

3. de Sa J, Mounier-Jack S, Coker R. Risk communication and management in public health crises. Public Health. 2009;123(10):643-644. doi: 10.1016/ j.puhe.2009.07.017.

4. Cope JR, Frost M, Richun L, et al. Assessing knowledge and application of emergency risk communication principles among public health workers in China. Disaster Med Public Health Prep. 2014;8(3):199-205. doi: 10.1017/dmp.2014.29. PMID: 24785302.

5. Radović V, Curčić L. The opportunities of crises and emergency risk communication in activities of Serbian public health workforce in emergencies. Iran J Public Health. 2012;41(10):15-23. PMID: 23308348.

\title{
Validation Study of the World Health Organization and Pan American Health Organization Hospital-Based Disaster Preparedness Questionnaires in Nepal
}

\author{
Ashma Baruwal, MPH; Pedro Arcos González, MD, PhD; Rafael Castro Delgado, MD, PhD; \\ Ganesh Kumar Jimee, MSc
}

$\mathrm{T}$ he World Disaster Reduction Campaign on Safe Hospitals has raised awareness of the need for hospitals and health facilities to remain safe and functional in disasters. ${ }^{1}$ Here we report the findings of our study that explored differences in results provided by 2 validated questionnaires in Nepal. Twin earthquakes recently hit the country, and the lack of preparedness among the majority of hospitals was evident in the response phase.

This cross-sectional study was carried between April 2014 and May 2014 in 9 hospitals that were included on the basis of highest patient flow. One selected hospital chose not to participate, citing lack of approval from their ethics committee. Interviews were conducted with hospital directors or chiefs of disaster response teams (wherever applicable), except for one hospital where the questionnaire was self-administered.

Functional aspects of hospital preparedness were studied by using the World Health Organization (WHO)-Europe and Pan American Health Organization (PAHO) validated questionnaires. While comparing functional aspects of the WHO and PAHO questionnaires, 8 components were identified as similar in nature. Post-disaster recovery was found only in the WHO questionnaire and as a result was not included in the analysis. Questionnaires were designed and well accepted to determine the functional ability of hospitals during a disaster and to identify areas that need improvement. $^{2,3}$ 
Taking WHO as the reference, questions from PAHO corresponding to the WHO questionnaire were grouped. Questions not similar on either questionnaire were not considered for analysis. Responses on the WHO questionnaire were categorized into 3 levels: due for review, under progress, and completed. Scores were assigned for the 3 categories of responses as follows: "due for review" as 1, "under progress" as 2 , and "completed" as 3. Responses on the PAHO questionnaire were categorized into 3 levels: low, average, and high. Likewise, scores were assigned for the 3 categories of responses on an ordinal level with "low" as 1, "average" as 2, and "high" as 3.

The results revealed no differences between the outcome of components of either questionnaires except for the command and control section $(P=0.002)$. This could be because the command and control component of the PAHO questionnaire had questions pertaining to a separate space, equipment, back-up systems for the Emergency Operations Center, whereas the WHO questionnaire had questions specific to the Hospital Incident Command organizational structure. However, this was not an absolute analysis because we were comparing only 2 similar questions and not the same ones.

Both checklists had questions on safe hospital evacuation. The WHO checklist had a continuous monitoring system to identify potential vulnerable areas such as entry/exits and food/water access points prior to any disaster. However, it was noticed during the interview that the respondents confused the related question with crowd control after the disaster, which required repeated explanation.

Descriptive statistics were used for analysis of the WHO questionnaire, whereas the PAHO questionnaire had its own safety scoring index with a safety calculator. ${ }^{4}$ The WHO questionnaire was user-friendly because it was easy to analyze. The PAHO questionnaire had its own safety scoring index with a scoring calculator that was not readily available.

We hope our comparison can help hospitals to select a proper evaluation tool, especially considering that the resources of hospitals in Nepal are limited.

\section{About the Authors}

Unit for Research in Emergency and Disaster, Department of Medicine, University of Oviedo, Oviedo, Spain (Ms Baruwal, Profs Arcos and Castro); the National Society for Earthquake Technology-Nepal, Kathmandu, Nepal (Mr Jimee).

Correspondence and reprint requests to Ashma Baruwal, MPH, Unit for Research in Emergency and Disaster, Department of Medicine, University of Oviedo, Oviedo, Spain (e-mail: baruwalashma@gmail.com).

Published online: March 30, 2016.

\section{REFERENCES}

1. World Health Organization. Safe hospitals and health facilities. WHO website. http://www.who.int/hac/techguidance/comprehensive_safe_ hospital_framework.pdf?ua=1. Published 2015. Accessed June 1, 2015.

2. Disaster Preparedness and Response. Hospital emergency response checklist. World Health Organization-Regional Office for Europe website. http://www.euro.who.int/_data/assets/pdf_file/0020/148214/e95978.pdf. Published 2011. Accessed June 18, 2015.

3. Emergency Preparedness and Disaster Relief. Hospital safety index. Organizacion Panamericana de la salud website. http://www.paho.org/ disasters/index.php?option $=$ com_content $\&$ view $=$ category $\&$ layout $=$ blog $\& i d=907 \&$ Itemid=884. Accessed June 18, 2015.

4. Disaster Preparedness and Response. WHO's hospital safety index and emergency checklist rolled out in Europe. World Health OrganizationRegional Office for Europe website. http://www.euro.who.int/en/healthtopics/emergencies/disaster-preparedness-and-response/news/news/2012/10/ whos-hospital-safety-index-and-emergency-checklist-rolled-out-in-europe. Published October 12, 2012. Accessed June 20, 2015.

\title{
doi:10.1017/dmp.2016.19
}

\section{Futuristic First Responders}

\author{
Zion Tsz Ho Tse, PhD; Sierra Hovet, BE; Chi-Ngai Cheung, MPhil; Isaac Chun-Hai Fung, PhD
}

$\mathrm{T}$ he ultimate sacrifice of 104 firefighters at the August 12, 2015, Tianjin explosions and the 10th anniversary of Hurricane Katrina call for the development of rescue robots as first responders. ${ }^{1,2}$ Robots can be designed to combat fires, contain chemicals, rescue citizens, and perform other dangerous tasks. During disasters, the appropriate use of rescue robots could save lives.

The Defense Advanced Research Projects Agency (DARPA) Robotics Challenge (DRC) is an international competition that encourages the development of robots for performing rescue tasks in disaster zones. Some key elements of rescue robots that need further improvement are the ability to act autonomously, the mode and reliability of remote operation, the limitations of power cords, the ability to get in and out of vehicles, and dexterity that does not compromise robustness, strength, mobility, and balance. ${ }^{3}$

Because disaster relief tasks require operating equipment and maneuvering in buildings designed for humans, most rescue robots have human-like outlook and functionalities. Atlas is a DARPA-funded project to develop humanoid robots to serve 